\title{
La educación ambiental y el desarrollo sostenible en el contexto colombiano
}

\author{
Environmental Education and Sustainable Development in the Colombian Context

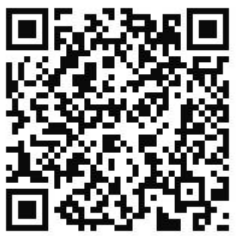 \\ Gloria Yaneth Flórez-Yepes ${ }^{1}$ \\ Universidad de Manizales \\ Manizales, Colombia \\ gflorez@umanizales.edu.co
}

Recibido 16 de mayo de 2014 • Corregido 6 de mayo de 2015 • Aceptado 31 de julio de 2015

Resumen. El objetivo de esta discusión es hacer un análisis de la educación ambiental en Colombia y su contexto frente al desarrollo sostenible, considerando la forma de abordaje del tema de la sostenibilidad como un proceso de inclusión de las instituciones educativas y con base en los lineamientos de política nacional. Se resalta, además, la importancia del abordaje de la educación ambiental en un contexto de la participación social como una forma de apropiación y acción; en este sentido, metodológicamente para el desarrollo del análisis se retoman algunas bases conceptuales para, desde allí, plantear una postura analítica y crítica. Para su análisis se consideran cuatro aspectos importantes: i) el desarrollo sostenible desde su concepto y evolución, ii) el concepto de sostenibilidad en la educación, iii) la educación ambiental en Colombia, su evolución e impactos y iv) la investigación acción participativa como una estrategia importante para el abordaje de los procesos de educación ambiental. Se obtienen las siguientes conclusiones: i) es necesario hacer análisis de los impactos que han generado los procesos de educación ambiental en Colombia, para determinar si las formas de abordaje han sido las más efectivas o si, por el contrario, se necesita repensar y promover nuevos escenarios para hacer procesos de transformación efectivos; ii) la educación ambiental es un reto de todos y todas que se debe manejar de forma sistémica e integral. Con el análisis se concluye que, a pesar de los grandes esfuerzos realizados en Colombia frente a la educación ambiental, el país aún adolece de la problematización y adopción de una educación para el desarrollo sostenible, que permita garantizar una formación integral que involucre, de manera sistémica, todos los escenarios que comprende la sostenibilidad.

Palabras claves. Educación ambiental, desarrollo sostenible, investigación acción participativa, procesos de transformación.

${ }^{1}$ Administradora ambiental, Magister en Desarrollo Sostenible y Medio Ambiente, estudiante de doctorado en Desarrollo Sostenible, Universidad de Manizales. Docente Universidad Católica de Manizales y docente Universidad de Manizales. 
doi: http://dx.doi.org/10.15359/ree.19-3.5

URL: http://www.una.ac.cr/educare

CORREO: educare@una.cr

\begin{abstract}
The aim of this discussion is to analyze environmental education in Colombia and its context in view of sustainable development, considering how to approach sustainability as a process of inclusion of educational institutions and based on the guidelines of national policy. Further, it highlights the importance of addressing environmental education in a context of social participation as a form of appropriation and action; in this sense, some conceptual bases are methodologically taken up and, from that point, we will be able to establish an analytical and critical stance. Its analysis considers four important aspects: i) sustainable development from its concept and evolution, ii) the concept of sustainability in education, iii) environmental education in Colombia, its evolution and impacts and iv) participatory action research as an important strategy to address the environmental education processes. The following conclusions were obtained: i) it is necessary to analyze the impacts generated by environmental education processes in Colombia, to determine whether its approach has been the most effective or if, on the contrary, there is a need to rethink and promote new scenarios for effective transformation processes; ii) environmental education is a challenge for all and it must be managed systemically and comprehensively. The analysis helps us to conclude that despite the great efforts that have been made in Colombia regarding environmental education, the country is still suffering from the problematization and adoption of an education for sustainable development that will allow for comprehensive training systemically involving all the scenarios that comprise sustainability.
\end{abstract}

Keywords. Environmental education, sustainable development, participative action research, transformation processes.

Hablar de la educación ambiental y el desarrollo sostenible debe constituir un direccionamiento de enseñanza y aprendizaje, el cual incluya una formación integral que debe estar soportada en los valores y principios, pero sobre todo desde una concepción ética.

Podría entenderse, entonces, que la formación integral deba implicar una estrategia de aprendizaje intencionada, tendiente al fortalecimiento de una personalidad responsable, con capacidad de reconocer e interactuar con su entorno; es preciso, por tanto, que sobre este concepto de integralidad la educación para el desarrollo sostenible asuma una postura que incluya el reconocimiento de las problemáticas ambientales y la necesidad de aprender a interactuar con el medio ambiente. Desde ese concepto de educación para el desarrollo sostenible debe, principalmente, propiciarse una actuación del estricto marco ético y moral:

La ética, como rama de la filosofía, se enfoca al estudio y evaluación de las conductas o comportamientos humanos, a la luz de principios morales y valores, considerando las acciones respecto a lo que es correcto o no, a lo que es bueno o malo, tomando en cuenta los motivos y fines de tales acciones. (Estrada, Monroy y Ramírez, 2005, p. 30)

La ética ha evolucionado a través de los siglos conforme el ser humano ha reflexionado sobre las intenciones y consecuencias de sus actos. De esas reflexiones sobre la naturaleza del comportamiento humano, ha surgido la necesidad de desarrollar una mayor y mejor conciencia 
doi: http://dx.doi.org/10.15359/ree.19-3.5

URL: http://www.una.ac.cr/educare

CORREO: educare@una.cr

que dé dirección ética al pensamiento y a la acción humana; en este sentido, una de las causas fundamentales de las problemáticas ambientales mundiales es la debilidad o ausencia de valores. No se puede lograr una educación para el desarrollo sostenible, si no se incorporan como principios fundamentales los componentes éticos y de valores humanos.

Partiendo de lo anterior, en este ensayo se pretende hacer una reflexión y análisis del desarrollo sostenible y la educación ambiental en Colombia, un país que se encuentra en un proceso de construcción de paz y que, por lo tanto, debe incluir proyectos que, además de asegurar la disminución de la desigualdad, también involucren las acciones encaminadas a generar, en la ciudadanía, una formación en valores éticos, que garanticen la responsabilidad, el conocimiento y la capacidad para identificar y enfrentar conjuntamente la solución a las problemáticas ambientales, y promover la participación social y la educación ambiental.

Finalmente, este ensayo comprende cuatro temas importantes para su análisis: los conceptos de desarrollo sostenible y de la sostenibilidad a la educación; la educación ambiental en Colombia y la investigación acción participativa como herramienta de la educación para el desarrollo sostenible; los cuales son fundamentales para el entendimiento de la inclusión de un nuevo modelo de educación ambiental que permita prepararnos para una nueva tendencia de desarrollo.

\section{El desarrollo sostenible}

El concepto de desarrollo sostenible ha sido interpretado de diferentes maneras desde que fue concebido por el informe del Brundtland por la Comisión Mundial sobre el Medio Ambiente y Desarrollo en 1987, que expresa que el desarrollo sostenible es aquel que satisface las necesidades del presente sin comprometer la capacidad de las futuras generaciones de satisfacer sus propias necesidades (Riechmann, 1995). Ha sido un concepto revolucionario en la medida que para entenderlo e interiorizarlo es necesario involucrar los componentes de ambiente, economía y sociedad; de acuerdo con el Documento de Orientación Política, Cumbre Mundial de Líderes Locales y Regionales en el 3er Congreso Mundial (CGLU, 2010), desde el año 2010 la cultura se involucra como un cuarto pilar en este concepto, pues se considera como la que determina la forma de actuar de las personas en el mundo frente al desarrollo sostenible.

Según lo anterior, la UNESCO, World Conference on Education for Sustainable Development (2009), en el proyecto de Educación sobre el Patrimonio para el Desarrollo Sostenible, pretende reforzar los lazos entre la cultura y la educación para el desarrollo sostenible, cuyo propósito es hacer inventarios del patrimonio material e inmaterial, para que a través de propuestas participativas sean tomadas decisiones conjuntas que contribuyan a generar soluciones ambientales futuras: definitivamente, el componente cultural es fundamental en la toma de decisiones ambientales. 
doi: http://dx.doi.org/10.15359/ree.19-3.5

URL: http://www.una.ac.cr/educare

CORREO: educare@una.cr

En este sentido, la política nacional de educación ambiental dentro de sus criterios menciona la interculturalidad y la diversidad cultural, el intercambio y el diálogo entre diferentes culturas como un aspecto fundamental para abordar el concepto de educación ambiental.

Por otro lado, (Gallopin, 2003) plantea que el desarrollo sostenible debe verse desde una visión sistémica, puesto que es un enfoque que puede proporcionar una perspectiva más útil que otras formas de análisis. No se puede pensar en los conceptos que integran la sostenibilidad de una manera independiente, pues el éxito o fracaso del desarrollo sostenible depende de la forma como se integren estos componentes para enfrentar los retos del desarrollo económico y la forma como se aborden los problemas ambientales y sociales.

Gallopin (1994) propone un conjunto de atributos necesarios para la sostenibilidad del sistema como un todo; dentro de los cuales se consideran los siguientes: la disponibilidad de recursos, la capacidad de adaptación y flexibilidad para entender y detectar los cambios que pasan en el mundo exterior; homeóstasis general relacionada con la resiliencia y estabilidad; $y$ la capacidad de respuesta que tiene que ver con la capacidad del sistema socioecológico para hacer frente al cambio.

Ahora bien, el llamado capital natural no es sustituible por ningún tipo de capital humano, antes bien, son complementarios (Daly, 1997), ambos intervienen en la evolución de una sociedad.

En esta búsqueda de conceptos de la sostenibilidad se han podido diferenciar dos tipos de tendencias: la sostenibilidad débil y la sostenibilidad fuerte; la primera, podría definirse, según Luffiego y Rabadán (2000), como la viabilidad de un sistema socioeconómico en el tiempo; mientras que la segunda se puede definir como la viabilidad de la relación que mantiene un sistema socioeconómico con un ecosistema (Naredo, 1994).

El concepto de medio ambiente que subyace a esta concepción de sostenibilidad es sistémico y global. El sistema socioeconómico se encuentra integrado en el ecosistema, es un sistema abierto, continuamente está entrando materia y energía (recursos) en él y saliendo desechos; la viabilidad de esta interacción depende del mantenimiento de los procesos del ecosistema. (Luffiego y Rabadán, 2000, p. 476)

A raíz de la cumbre de Río nace el concepto de la sostenibilidad integral, y a pesar de que hay varias interpretaciones y matices de este concepto, donde se establecen diferencias entre la sostenibilidad ecológica, social y económica, algunos estudios consideran que tales tipos de sostenibilidad constituirán las dimensiones bajo un único concepto; sin embargo, el concepto es importante en la medida en que se pueda materializar en la práctica, pues es claro que no existe una integración de las diferentes dimensiones. Esta integración debería estar reflejada desde las mismas decisiones políticas y desde un abordaje del trabajo interdisciplinario que permita tener visiones objetivas frente a las acciones y decisiones para generar verdaderos procesos de sostenibilidad. 
doi: http://dx.doi.org/10.15359/ree.19-3.5

URL: http://www.una.ac.cr/educare

CORREO: educare@una.cr

El término "desarrollo sostenible", como se ha mencionado en esteescrito, tiene un significado muy amplio, pues implica un conjunto de cambios en la estructura económica, social, institucional y política de los distintos países del mundo. Es sinónimo de mejora, de progreso; indica un cambio hacia una situación preferible a la actual, que conduce a una transformación positiva.

\section{Introducción del concepto de sostenibilidad a la educación}

No se puede pretender pensar en los cambios de conciencia social frente a los recursos naturales de maneras individuales, por lo tanto, la toma de conciencia ambiental debe ser un proceso colectivo que permita la solución o mitigación de los grandes impactos ambientales que se están presentando a nivel mundial y que además ha traído consecuencias sociales significativas. En este sentido, la educación juega un papel importante, pues a través de ella se puede lograr encontrar un camino que permita entender los problemas ambientales globales; pero que, a la vez, contribuya a generar habilidades que posibiliten encontrar soluciones conjuntas desde una visión de futuro.

De acuerdo con lo anterior, la UNESCO, World Conference on Education for Sustainable Development (2009) en la declaración de Bonn, invita a la realización de diferentes acciones para que el desarrollo sostenible imprima una nueva dirección a la enseñanza y el aprendizaje para todas las personas, promueva la educación de calidad e integre a todos y todas, sin excepción, basándose en valores, principios y prácticas necesarios para responder eficazmente a los retos actuales y futuros; con capacidad de enfrentar los problemas asociados al agua, la biodiversidad, la generación de energía, el cambio climático, la mitigación y adaptación del riesgo y los desastres, la pérdida de la biodiversidad, la crisis alimentaria, las amenazas contra la salud, la vulnerabilidad social y la inseguridad, entre otros.

Un aspecto importante que menciona la declaración es que aún se siguen teniendo múltiples interpretaciones y concepciones de la educación para el desarrollo sostenible, $y$, en este sentido, no ha sido posible lograr verdaderas acciones que contribuyan a cumplir con sus objetivos; de acuerdo con esto, también se considera la desigualdad como un factor determinante para el incumplimiento de las metas establecidas. Pasará, sin duda, mucho tiempo hasta que se pueda llegar a cumplir con los diferentes ítems determinados para el llamamiento a la acción, establecidos en esta declaración.

La visión de la educación para el desarrollo sostenible debe implicar:

Una transformación educativa que va desde la modificación de la estructura, la gestión, los currículos y en los espacios y estrategia de formación y aprendizaje, es decir, no sólo un cambio en los contenidos sino un cambio sistémico. (Macedo y Salgado, 2007, p. 35) 
doi: http://dx.doi.org/10.15359/ree.19-3.5

URL: http://www.una.ac.cr/educare

CORREO: educare@una.cr

La educación debe convertirse en un espacio donde sus aulas se conviertan en verdaderos centros de producción de conocimiento, donde se prepare tanto a docentes como estudiantes para un cambio de comportamiento, donde se integren los principios de sostenibilidad, los cuales deben convertirse en lineamientos de vida.

En Colombia, la inmersión del concepto de sostenibilidad dentro de la educación ambiental aún sigue siendo incipiente; sin embargo se han diseñado diferentes estrategias para fortalecer la integralidad de la educación ambiental a partir del desarrollo de proyectos PRAE (proyectos ambientales escolares), los cuales tienen como objetivo involucrar a toda la comunidad educativa en un proceso de educación ambiental, pero a pesar de que estos han evolucionado, aún falta fortalecer y apropiar el concepto de sostenibilidad desde todas sus dimensiones, (ambiental, económica, social, cultural) con el fin de poder llegar a una educación para el desarrollo sostenible.

\section{La educación ambiental en Colombia}

De acuerdo con Guillén (1996) “la Educación Ambiental se concibe como una dimensión que debe integrarse en las propuestas educativas dirigidas a la sociedad" (p. 106), la UNESCO, World Conference on Education for Sustainable Development (2009) plantea que en la educación ambiental se deben reconocer valores, aclarar conceptos y fomentar actitudes y aptitudes, con el fin de comprender y apreciar las interrelaciones entre el hombre, la cultura y el medio.

En Colombia se han venido introduciendo algunas acciones de educación ambiental a partir de los años 70 desde la conferencia de Estocolmo, cuyo propósito ha sido preparar al ser humano para el desarrollo, desde una mirada de protección al medio ambiente. En este sentido, "entre 1998 y 2002, en el marco de la Política Ambiental del Plan de Desarrollo: 'Cambio para construir la paz', el Ministerio del Medio Ambiente diseña y pone en ejecución el Proyecto Colectivo Ambiental, el cual se posiciona como su carta de navegación" (MINAMBIENTE, MINEDUCACIÓN, 2002, p. 7). Este proyecto determina las acciones encaminadas a generar, en la ciudadanía, criterios de ética, responsabilidad, conocimiento y la capacidad para identificar y enfrentar conjuntamente la solución de los problemas ambientales, dándole vital importancia a la participación y la educación ambiental.

Han sido muchos los debates que se han dado en el país frente al manejo que se le ha dado al tema de la educación ambiental; sin embargo, desde la concepción política y desde los tomadores de decisiones, se empezaron a preguntar si en Colombia se llevan cerca de 20 años desarrollando iniciativas para la educación ambiental, ¿por qué no se cuenta hoy con un cambio social frente al medio ambiente? ¿Cómo se han enfocado las acciones hacia la educación ambiental? ¿Cuáles son los obstáculos que existen para llegar a formar los nuevos ciudadanos y ciudadanas que requiere el país? (Torres, 1998).

6 
Torres (1998) acota:

Puesto que para estas preguntas no se tenían respuestas claras sino supuestos e hipótesis, y tomando en cuenta el propósito que se quería lograr... se pensó que lo más urgente y adecuado no era la formulación de un plan nacional de Educación Ambiental sino, más bien, conocer el país e intentar comprender cómo pensaba la gente, cómo actuaba, cómo estaba desarrollado sus procesos, qué conceptualizaciones estaban moviendo a la Educación Ambiental, qué estrategias y metodologías se estaban construyendo, y hasta qué punto la escuela estaba implicada en todos estos procesos. (p. 26)

Esta reflexión conduce a hacer un alto en el camino, pues no se puede pensar en una educación ambiental encaminada a desarrollar acciones que conlleven al cumplimiento de indicadores nacionales. La educación ambiental se debe construir desde un pensamiento integral que considere las particularidades de las personas; que no se conciba solo desde las actividades escolares donde los educadores y las educadoras aún no tienen convencimiento de sus principios para poderlos fomentar en el estudiantado; debe ser una educación ambiental pensada desde las concepciones culturales, familiares; pero, sobre todo en Colombia, se empezó a pensar en que lo importante para la educación ambiental era que hubiera un sentido de pertenencia por su entorno, por parte de las comunidades, pues mientras que no hubiera participación comunitaria en los proyectos ambientales ni sentido de apropiación hacia sus ecosistemas, no se podría pensar en tener un proceso de transformación de la educación ambiental en el país.

De acuerdo con Torres (1998), el programa de educación ambiental para Colombia (decreto 1743 de 1994) está concebido en cuatro etapas fundamentales: la etapa de exploración, en la que se identificaron los actores comprometidos con el tema en Colombia; la etapa de profundización, donde se hace toda la conceptualización de la educación ambiental; la etapa de proyección, donde se busca a través de la concertación poder llegar a acuerdos efectivos de educación ambiental para el país, y la etapa de estrategias, en la que se definen los lineamientos y criterios sobre los cuales se debe abordar la educación ambiental.

El Proyecto Ambiental Escolar (PRAE) ha sido, en el país, una estrategia pedagógica que ha pretendido la comprensión de la problemática ambiental local y contribuye en la búsqueda de soluciones acordes con las realidades de cada región y municipio, en un contexto natural, social, cultural, político y económico. La intención de los PRAE, según el MinEducación (2005), debe involucrar:

A todos los miembros de la comunidad educativa, instituciones del sector y organizaciones sociales, mediante la integración de conocimientos y experticias en torno a un objetivo: interpretar un problema ambiental concreto y participar en la búsqueda de soluciones, desde una gestión ambiental sostenible. (párr. 4) 
doi: http://dx.doi.org/10.15359/ree.19-3.5

URL: http://www.una.ac.cr/educare

CORREO: educare@una.cr

Sin embargo, a pesar de que esta ha sido una buena intención, se ha convertido, en muchas ocasiones, en una estrategia activista en la cual no necesariamente participa toda la comunidad educativa, sino que se centra en proyectos concretos dentro de la institución donde participan no más que el personal docente encargado y el alumnado, dejando de lado los demás actores de la comunidad educativa; por otro lado, estos proyectos ambientales escolares se han dirigido especialmente a dar soluciones a problemáticas asociadas con el reciclaje dentro de las instituciones $y$, en muchas ocasiones, se ha dejado de lado el abordaje de las demás problemáticas ambientales que no se tienen en cuenta, ya sea por el desconocimiento de su existencia o por el facilismo de cumplir con los indicadores de cada una de las instituciones. En este sentido, la intención que se ha tenido desde el MEN ha sido buena, frente al abordaje que quiere hacer de los procesos de educación ambiental; sin embargo, las aplicaciones locales de las estrategias aún deben ser muy fortalecidas para que se tengan verdaderos procesos de transformación e impacto que aseguren la participación de todos los actores y se inicie con un verdadero proceso de apropiación de la necesidad de entender la problemática ambiental actual y buscar soluciones a esta.

Por otro lado, la educación ambiental en Colombia se ha incluido dentro del currículo escolar desde los primeros años escolares, la cual ha sido fortalecida desde las asignaturas de ciencias naturales; sin embargo, en la educación superior, la educación ambiental ha sido una temática indispensable dentro de la maya curricular de cada programa, incluyéndose dentro de las asignaturas obligatorias exigidas por el gobierno nacional; sin embargo, es importante mencionar que ha hecho falta realizar análisis de los impactos que ha tenido la inclusión de estas asignaturas dentro del pensum académico que permitan fortalecer los procesos o realizar cambios estructurales para garantizar que esta temática se aborde de manera transversal al proceso académico y se pueda responder a la necesidad de una formación más integrada.

\section{La investigación acción participativa como herramienta de la educación para el desarrollo sostenible}

LaIAP esuna metodología importante, que permite no solola apropiación del conocimiento, sino genera respuestas concretas a problemáticas planteadas por investigaciones que abordan problemáticas reales y desean aportar alguna alternativa de cambio o transformación. Martínez 2009 (p. 28, retomado por Colmenares, 2012, p. 104) afirma: "el método de la investigaciónacción tan modesto en sus apariencias, esconde e implica una nueva visión de hombre y de la ciencia, más que un proceso con diferentes técnicas".

La investigación-acción participativa permite que exista una integración del conocimiento y la acción, promueve que los actores puedan ser parte de los procesos, conozcan, interpreten y transformen la realidad objeto del estudio, a partir de un proceso de apropiación del entorno, 
doi: http://dx.doi.org/10.15359/ree.19-3.5

URL: http://www.una.ac.cr/educare

CORREO: educare@una.cr

este método facilita proponer alternativas de solución a las problemáticas identificadas por los propios actores sociales, donde el fin principal será generar cambios y transformaciones definitivas. La transformación y emancipación constituyen los ejes direccionadores de esta opción metodológica (Colmenares 2012).

Lo procesos de participación social hacen que la educación ambiental no se dimensione solo desde la educación formal y no formal, sino que involucre a toda la comunidad en procesos de transformación; sin embargo, es necesario también considerar que los procesos de transformación exigen no solo la participación activa sino también se requieren de tiempo para generar procesos de adaptación y actitud de cambio, en lo cual intervienen múltiples variables que tienen que ver con la cultura, el medio y demás factores que contribuyen a que, de manera integral, se pueda llegar a soluciones concretas de las problemáticas ambientales.

De acuerdo con Urrego (2009, p. 154):

En la cultura de cada sociedad existe un proceso formativo, costumbres y normas transmitidas de generación en generación, desde las cuales las instituciones educativas, con ellas el docente, tienen que trazar acciones que respondan a las necesidades personales, sociales y profesionales en este contexto.

Si bien la escuela favorece el escenario para la participación de todas las partes: docentes, estudiantes, padres y madres de familia, también es cierto que el entorno social es fundamental para favorecer los procesos de construcción; el papel docente es preponderante para estos procesos, pues es quien podría lograr compenetrarse con un compromiso de cambio, a la vez que oriente sus procesos de formación hacia la protección y cuidado del medio ambiente.

Por otro lado, desde los mismos procesos de formación se ha venido trabajando en la construcción de políticas contundentes que conlleven al fortalecimiento de la educación ambiental, pues indiscutiblemente, este es un tema que no puede abordarse desde las intenciones locales, sino que, por el contrario, es un proceso generalizado que nos involucra a todos y a todas, y que debe direccionarse desde una visión política donde quienes toman las decisiones lo hagan con el convencimiento de la necesidad de generar verdaderos cambios que impacten y generen transformaciones.

\section{Conclusiones}

La cultura definitivamente es la mediadora para concluir verdaderos procesos de transformación social o cambios de comportamiento frente a la conservación del medio ambiente.

La educación ambiental o la educación para el desarrollo sostenible debe verse y abordarse desde una visión sistémica, donde se involucren todos los componentes de la sostenibilidad. 
doi: http://dx.doi.org/10.15359/ree.19-3.5

URL: http://www.una.ac.cr/educare

CORREO: educare@una.cr

Colombia ha avanzado frente a la forma de abordaje de la educación ambiental; sin embargo, en muchas ocasiones la política ha sido aplicada en los niveles locales desde una perspectiva activista que se ha limitado a acciones concretas sin mayores impactos.

La investigación acción participativa es una buena opción para el abordaje de la educación ambiental, pues a través de ella se permite hacer apropiación del conocimiento-acción, pero también posibilita los procesos de transformación.

La educación ambiental es un reto de todos y todas que no se logra en el corto plazo, es un proceso donde deben integrarse todos los escenarios para hacer posible la adaptación al cambio.

Es necesario que quienes tienen a cargo la toma de decisiones en los procesos de construcción de una nueva mirada hacia la educación ambiental se sigan haciendo permanentemente preguntas relacionadas con los logros, impactos, beneficios que se han generado con los esfuerzos hasta ahora realizados, con el fin de buscar nuevas alternativas que permitan mejorar y llegar a verdaderos procesos de educación ambiental.

Educación para el desarrollo sostenible debe ser un modelo de desarrollo basado en conocimiento desde la educación, innovación, y el trabajo en red tiene que avanzar a generar seres humanos para lo económico, lo social y lo ambiental; además, debe ser el puente para el fortalecimiento de la cultura y el soporte para la tendencia de una nueva forma cultural de respecto por los recursos naturales y una nueva forma de abordaje de estos.

\section{Referencias}

Ciudades y Gobiernos Locales Unidos (CGLU). (17 de noviembre de 2010). Documento de orientación política, cumbre mundial de líderes locales y regionales. En 3er Congreso Mundial de CGLU, México.

Colmenares, A. M. (2012). Investigación-acción participativa: Una metodología integradora del conocimiento y la acción. Voces y Silencios: Revista Latinoamericana de Educación, 3(1), 102-115. Recuperado de http://dialnet.unirioja.es/servlet/articulo?codigo $=4054232$

Daly, H. E. (1997). De la economía del mundo vacío a la economía del mundo lleno. En R. Goodland (Coord.), Medio ambiente y desarrollo sostenible: Más allá del informe Brundtland (pp. 37-50). Madrid:Trotta.

Estrada, R. A., Monroy, G. S. y Ramírez, H. T. (Diciembre, 2005). Ética-responsabilidad-socialdesarrollo sustentable en las organizaciones. Administración y Organizaciones, 8(15), 27-45. Recuperado de http://148.206.107.15/biblioteca digital/estadistica.php?id ho st=6\&tipo=ARTICULO\&id=2341\&archivo=9-155-2341 yny.pdf\&titulo=\%C3\%89ticaresponsabilidad\%20social-desarrollo\%20sustentable\%20en\%20las\%20organizaciones 
doi: http://dx.doi.org/10.15359/ree.19-3.5

URL: http://www.una.ac.cr/educare

CORREO: educare@una.cr

Gallopín, G. (2003). Sostenibilidad y desarrollo sostenible: Un enfoque sistémico (Serie medio ambiente y desarrollo 64). Santiago de Chile: Naciones Unidas. Recuperado de http:// repositorio.cepal.org/bitstream/handle/11362/5763/S033120 es.pdf?sequence $=1$

Gallopin, G. (1994). Impoverishment and Sustainable Development. A Systems Approach [Empobrecimiento y desarrollo sostenible]. Canadá: HSD. Recuperado de http://www.iisd. org/pdf/impoverishment_and_sd.pdf

Guillén, F. C. (Mayo-Agosto, 1996). Educación, medio ambiente y desarrollo sostenible. Revista Iberoameriana de Educación, 11, 103-110. Recuperado de http://www.oei.es/oeivirt/ rie11a03.pdf

Luffiego, M. y Rabadán, J. M. (2000). La evolución del concepto de sostenibilidad y su introducción a la enseñanza. Enseñanza de las Ciencias: Revista de Investigación y Experiencias Didácticas, 18(3), 473-486. Recuperado de http://www.raco.cat/index.php/Ensenanza/article/ view/21701/21535

Macedo, B. Y Salgado, C. (2007). Educación ambiental y educación para el desarrollo sostenible en América Latina. Revista Forum de Sostenibilidad. 1, 29-37. Recuperado de http://www. ehu.eus/cdsea/web/revista/numero_1/01_03macedo.pdf

Ministerio del Medio Ambiente (MINAMBIENTE) y Ministerio de Educación Nacional (MINEDUCACIÓN). (2002). Política nacional de educación ambiental Sina. Bogotá: Autor. Recuperado de http://webcache.googleusercontent.com/search?q=cache:l1zUzPxwXto J.cmap.upb.edu.co/rid\%3D1195259861703_152904399_919/politi-ca_educacion_amb. $\mathrm{pdf}+\& \mathrm{~cd}=1 \& \mathrm{hl}=\mathrm{es} \& \mathrm{ct}=\mathrm{clnk}$

MinEducación. (Agosto-Setiembre de 2005). Educación ambiental construir educación y país. Altablero, 36. Recuperado de http://www.mineducacion.gov.co/1621/article-90891.html

Naredo, J. M. (1994). Fundamentos de la economía ecológica. En F. Aguilera y V. Alcántara (Comps.), Delaeconomía ambiental alaeconomíaecológica (pp.231-252). Barcelona:ICARIA y FUHEM. Recuperado de http://www.fuhem.es/media/ecosocial/File/Actualidad/2011/ LibroEA EE.pdf

Riechmann, J. (1995). Desarrollo sostenible: La lucha por la interpretación. En J. Riechman, J. M. Naredo, R. B. Gómez, A. Esteban, C. Taibo, J. C. Rodríguez y J. Nieto (Auts.), De la economía a la ecología (pp. 11-36). Madrid: Trotta. 
doi: http://dx.doi.org/10.15359/ree.19-3.5

URL: http://www.una.ac.cr/educare

CORREO: educare@una.cr

Torres, M. (1998). La educación ambiental: Una estrategia flexible, un proceso y unos propósitos en permanente construcción. La experiencia de Colombia. Revista Iberoamericana de Educación, 16, 23-48. Recuperado de http://www.rieoei.org/oeivirt/rie16a02.pdf

UNESCO, World Conference on Education for Sustainable Development. (31 de marzo a 2 abril, 2009). World Conference on Education for Sustainable Development. Bonn Declaration [Conferencia Mundial sobre educación para el desarrollo sostenible. Declaración de Bonn]. Bonn, Germany: Autor. Recuperado de http://unesdoc.unesco.org/ images/0018/001887/188799e.pdf

Urrego, A. J. (Mayo-Agosto, 2009). La investigación acción participativa en el contexto socioeducativo: Estrategia metodológica en la producción del conocimiento para la acción social. Revista Educare, 13(2), 154-166. Recuperado de http://revistas.upel.edu.ve/ index.php/educare/article/view/236/146

\section{Cómo citar este artículo en APA:}

Flórez-Yepes, G. Y. (Setiembre-Diciembre, 2015). La educación ambiental y el desarrollo sostenible en el contexto colombiano. Revista Electrónica Educare, 19(3), 1-12. doi: http://dx.doi.org/10.15359/ree.19-3.5

Nota: Para citar este artículo en otros sistemas puede consultar el hipervínculo "Como citar el artículo" en la barra derecha de nuestro sitio web: http://www.revistas.una.ac.cr/index.php/EDUCARE/index 